Document downloaded from:

http://hdl.handle.net/10251/65384

This paper must be cited as:

Boronat, P.; Perez Francisco, M.; Tavares De Araujo Cesariny Calafate, CM.; Cano Escribá, JC.; Manzoni, P. (2014). Evaluating Metrics for Optimal Path Selection in Large Wireless Community Networks. 11th ACM International Symposium on Performance Evaluation of Wireless Ad Hoc, Sensor, and Ubiquitous Networks (PE-WASUN 2014). ACM. doi:10.1145/2653481.2655235.

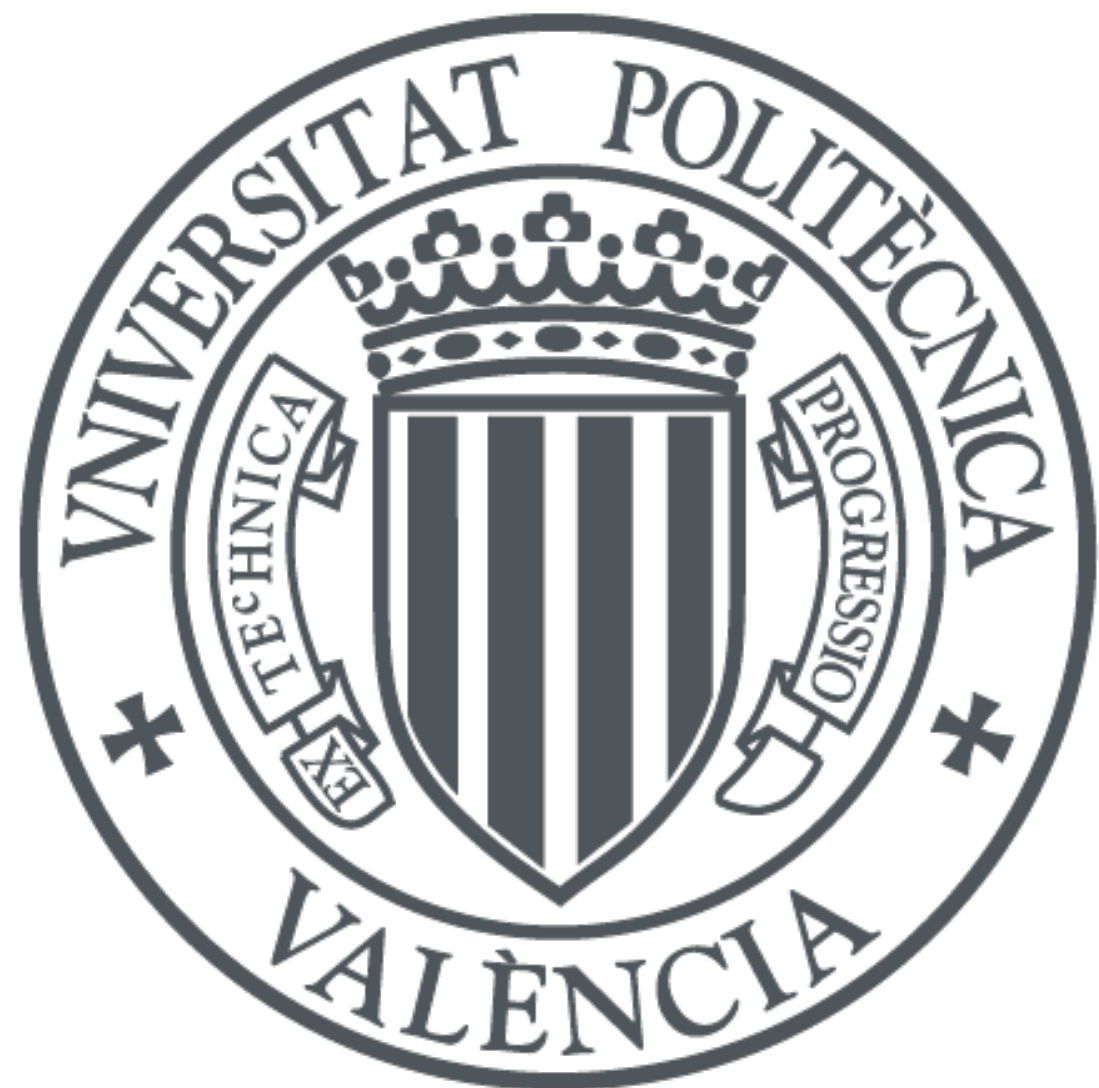

The final publication is available at

http://dl.acm.org/citation.cfm?doid=2653481.2655235

Copyright ACM

Additional Information

CACM 2014. This is the author's version of the work. It is posted here for your personal use. Not for redistribution. The definitive Version of Record was published in ACM, In Proceedings of the 11th ACM symposium on Performance evaluation of wireless ad hoc, sensor, \& ubiquitous networks (pp. 85-92). .http://dx.doi.org/10.1145/2653481.2655235 . 


\section{Evaluating Metrics for Optimal Path Selection in Large Wireless Community Networks}

\author{
Pablo Boronat \\ DLSI, Universitat Jaume I \\ Castellón, SPAIN \\ boronat@uji.es
}

\author{
Miguel Perez Francisco \\ DICC, Universitat Jaume I \\ Castellón, SPAIN \\ mperez@uji.es
}

\author{
Carlos T. Calafate \\ DISCA, Universitat Politècnica \\ de València, Spain \\ Valencia, SPAIN \\ calafate@disca.upv.es
}

\author{
Juan-Carlos Cano \\ DISCA, Universitat Politècnica \\ de València, Spain \\ Valencia, SPAIN \\ jucano@disca.upv.es
}

\author{
Pietro Manzoni \\ DISCA, Universitat Politècnica \\ de València, Spain \\ Valencia, SPAIN \\ pmanzoni@disca.upv.es
}

\begin{abstract}
Routing metrics commonly used nowadays in large wireless community networks (WCN), do not seem appropriate since they are generally not based on the real link capacity but on choosing the shortest path.

Routing protocols such as OSPF or BGP use static metrics, while wireless links should be evaluated frequently because their bandwidth varies dynamically. Ad hoc routing protocols use more complex metrics, however they are better suited for sporadic or mobile nodes than for static nodes placed in house roofs to create the backbone infrastructure.

In this work, we compare several metrics which might be of interest in realistic WCN scenarios. To do this, we have performed routing simulations over real topologies extracted from a specific WCN, namely "guifi.net".

The routes obtained using different metrics are compared in terms of length, capacity, and alternative paths. We show that, when using metrics based on real link bandwidth, the amount of routes with low bandwidth could be halved and the capacity of the network could be increased up to a $25 \%$.
\end{abstract}

\section{INTRODUCTION}

Community networks have become available since WiFi technology became affordable and mature. After a boom ten years ago, only the strongest communities persist and some of them experiment a sustainable growth. Although these networks can use various technologies, they are generally based on wireless links, given its low cost of deployment.

As supporters and active managers of one of the largest wire- less community networks, guifi.net ${ }^{1}$, we are continuously working on improving its performance. The wireless part of guifi.net, which connects most of the users, is mainly configured in infrastructure mode, meaning that routers are connected by using point-to-point links. Moreover, routers have also antennas available to provide coverage to client nodes. These routers use classical routing protocols, mostly OSPF and BGP. However, we have observed that these protocols are not well adapted to large wireless networks, basically for two reasons. First the metrics they use are just based on an administrative value calculated from the specific type of network device. With this procedure, all wireless devices are assigned the same cost, and the consequence is that the metric is reduced to the number of hops. This can be acceptable in wired networks (with controlled and stable bandwidth), but not when wireless links are involved, due to their very different and dynamic bandwidths.

The second basic issue of these protocols is that they only use the best routes (regarding the metric in use), and so routes can be easily congested under high traffic even when other acceptable routes are available. We believe that these drawbacks, specific to WCN, have important consequences on the overall network performance as the best links are not always used, and traffic is just concentrated in few routes.

In this paper we perform a thorough evaluation of various routing metrics in order to quantify the magnitude of this phenomenon. To achieve our goal, we establish a comparison among four path metrics used by different algorithms. The considered path metrics are the following:

- Bandwidth. In this case, the metric of a path represents its bandwidth or capacity. The metric for a path is the minimum bandwidth of all its links.

- Number of hops. This one is equivalent to current OSPF or BGP implementations.

- A combination of bandwidth and number of hops. This metric is calculated accumulating the inverse of the

\footnotetext{
${ }^{1}$ http://guifi.net
} 
real bandwidth of each component of the path. This solution resembles what could be generally considered as a good OSPF implementation.

- A metric called shortest widest bandwidth. In this case the best paths are those having the best bandwidth with the smaller number of hops.

It must be noticed that our focus in this work is not on how the components of routing metrics (i.e. link's bandwidth) used by our algorithms are obtained but on the impact that those metrics will have on performance. Our objective is to predict the benefits of using different metrics and path selection algorithms.

These path metrics are evaluated and compared through three criteria. The first one is the bandwidth or capacity; that is, the bandwidth between an origin and a destination if all resources are available for these two nodes. The second is the path hops count of the routes, which is related to the latency. Finally, we analyse the distribution function of the routes' bandwidth. This function provides more insight than the average path bandwidth. For instance, it is important to check the results for the worse selected paths, since network nodes under the acceptable bandwidth could be useless.

To evaluate the different path metrics, we use three real topologies extracted from the guifi.net database. From these topologies we estimate the (asymmetric) bandwidth of links depending on their length as well as the density of nodes in the respective area. Then the routes using the different path metrics are calculated in a routing simulator which has been developed for this work ${ }^{2}$. The routes used in the evaluation are those from each router of the network to a subset of nodes who provide shared Internet access.

We experimentally found that the most important result is that all metrics based on bandwidth probing avoid pathological cases. We also found that the best bandwidth metric is quite effective, but it produces a lot of best-cost paths for the same origin and destination nodes. In fact few of these paths are disjoint. Also, for these real topologies, the number of disjoint best-cost routes is not a significant comparison criteria, as all metrics have similar results.

The rest of this paper is organized as follows. The next section provides a brief description of related work. In Section 3 we present the scenario used for the simulation tests. Sections 4 and 5 are devoted to a detailed description of the evaluated path metrics, how they are used by the routing protocols, and the performance metrics (i.e. how paths metrics are compared). In Section 6 the set of experiments and the results are presented. Finally, Section 7 presents the conclusions and discusses the direction for future work.

\section{RELATED WORK}

Routing path metrics is certainly one of the most relevant topics that have been discussed in the field of routing protocols. A lot of research has been devoted to this topic, where

\footnotetext{
${ }^{2}$ The routing simulator is programmed in Python and uses NetworkX software. It will be delivered with GNU free license. It is made available by the authors upon request.
}

many experimental designs have been tested. A remarkable classification can be found in [2]. However, given the stability typical of wired networks, most routing protocols in common use have simplified this issue and they tend to just use static values as routing metrics. In the case of unstable wireless links, some protocols specifically designed for this unstable networks use dynamic metrics based on values obtained in real time $([5])$.

A lot of work is devoted to Mobile Ad Hoc Networks; for instance, [7], [8], [9] or [10]. However, these networks have different constraints to large infrastructure mode networks with static and permanent nodes.

Works such as [4] and [10] have goals similar to ours. Nevertheless, the main purpose of this work is to test three methods to measure the capacity of links (link probing techniques). In our case, we suppose that we already know link's bandwidth and we want to know how to use these data in order to improve the overall performance of the network. A general review on bandwidth probing and estimation can be found in [6].

In [1], [11] it is proposed one of the metrics that has been simulated, the shortest widest path metric. This metric is proposed in the context of QoS extensions for OSPF. Although being a bi-dimensional metric, in [1] it is shown that the metric can be used with similar computation cost than a single dimension one.

\section{SCENARIO OVERVIEW}

This work is motivated by the routing problems we observe in the guifi.net community network. In effect, the poor routing metrics used by routing protocols such as OSPF or BGP is one of the main drawbacks; however, to the best of our knowledge this phenomenon has never been quantified. These protocols rely on routing metrics based on an static administrative cost to represent the bandwidth of a link, rather than their real (and dynamic) capacity. Thus, in this work, we compared path selection algorithms that use different routing metrics in order to evaluate the achievable benefits.

For this evaluation, we have adapted the OSPF routing protocol to work with different path metrics in a simulation environment. In addition, to make the scenario more realistic, topologies from the guifi.net network have been imported. All data about this network are available in its web site (http://guifi.net). Topology (i.e. nodes, links and geographical positions) are exported in a specific XML format called CNML (Common Network Markup Language) [3].

From these topologies, we are interested in nodes participating in the dynamic routing (in guifi.net jargon, these nodes are known as multi-radio nodes or supernodes). A subset of these nodes provide public shared Internet connections, usually through Squid web proxies, and known as sink nodes.

In the evaluation process, we compare the routes obtained with different path metrics applied to the OSPF protocol.

For the comparisons we have used the routes from each node to the sink nodes, as these routes are more representative of 
Table 1: Values used for link's bandwidth estimation.

\begin{tabular}{c||c}
\hline maxbandwidth & $80 \mathrm{Mbps}$ \\
\hline minbandwidth & $128 \mathrm{Kbps}$ \\
\hline maxdistance & $15 \mathrm{~km}$ \\
\hline maxdensity & 0.0012 nodes $/ \mathrm{m}^{2}$ \\
\hline
\end{tabular}

real network traffic.

To feed the routing simulator, we also need the bandwidth of each link. These data are not available in the CNML format, as they represent dynamic real-time values. We have estimated the bandwidth of a link as a function of its length and the density of the nodes around the destination node. The longer the link, the lower its capacity. Also, the greater the density, the lower its bandwidth, in order to represent noise effect. Thus, links can be assigned a different bandwidth in each direction if there are different nodes densities in each edge (we have considered that all are point-to-point links). The bandwidths we have used in this work have a similar standard deviation than the real measures we captured in a part of the network.

The link bandwidth was computed as follows. From practical observations, we consider a maximum bandwidth of 80 Mbps for a TCP connection using a channel of $20 \mathrm{MHz}$ at negligible distance; this value has been tested with the Iperf utility using current double polarity IEEE 802.11n devices. From this value, the following function is applied. Let $l$ $(l>0)$ be the link's length in $\mathrm{km}$ and $d$ the density of nodes around the destination node in an area of a radius of 100 meters, given that the influence of close wireless devices is very high.

bandwidth $=M A X($ maxbandwidth $*(1-(l /$ maxdistance + $d /$ maxdensity)), minbandwidth)

We consider that a link longer than maxdistance or a density of nodes higher than maxdensity, has the minimum bandwidth (minbandwidth). For other cases, the bandwidth is reduced from the maximum observed bandwidth, maxbandwidth. The values we have used to estimate link's bandwidth have been obtained from a group of 34 routers in a guifi.net zone and they can be seen in Table 1. These constants provide similar average and standard deviation than our sample.

\section{ROUTING PATH METRICS}

In this section we explain the proposed routing path metrics. The simulated routing algorithms are based in the algorithm used by OSPF protocol. We have applied minor modifications in order to adapt this algorithm to each particular path metric.

One of the used metric is two-dimensional. Multidimensional metrics are rarely used in practice due to their high computation cost. However in [1] it is shown that the algorithm used in OSPF can be adapted to apply this metric with similar computation effort.
Among the considered metrics, traffic load has been discarded to prevent routing instability.

\subsection{Definitions}

We consider a topology or directed graph, $G(n, l)$ as a set of nodes and a set of directional links. Nodes represent the routers of a network and they are located geographically. A link connects two nodes, and it represents 1-hop connectivity between neighbouring routers. In this work it is assumed that the number of nodes of a topology is finite, and that from one node to another there is at most one directed link.

Each directional link has a starting node and an arriving node. Moreover, a directional link has a bandwidth. In a real network a link represents a direct hop between two routers, and its bandwidth could be measured. A (bidirectional) link is the composition of two directional links connecting the same nodes in both directions. Directional links of a link can have different bandwidths. Given a link $l$, let be $\operatorname{start}(l)$, end $(l)$ and bandwidth $(l)$ respectively the starting node, the arriving node and the bandwidth of $l$ in this direction. Also, we assume that bandwidth $(l)>0$.

We define a path, $p$, from a node $n_{o}$ to a node $n_{d}, n_{o} \neq$ $n_{d}$, as an ordered set of directional links without loops; that is, $p\left(n_{o}, n_{d}\right)=\left\{l_{1}, l_{2}, \ldots, l_{n}\right\},(n \geq 1)$, where $\forall l_{i} \in p$, $\operatorname{bandwidth}\left(l_{i}\right)>0, \operatorname{start}\left(l_{1}\right)=n_{o}, \operatorname{end}\left(l_{n}\right)=n_{d}$ and $\forall i$ $\left(1<i^{\natural} n\right), \operatorname{start}\left(l_{i}\right)=\operatorname{end}\left(l_{i-1}\right)$, and $\forall l_{i}, l_{j} \in p, i \neq j$, $\operatorname{start}\left(l_{i}\right) \neq \operatorname{start}\left(l_{j}\right)$.

Note that paths are also directional, and that from one node to another may exist more than one path. Next we define the four different metrics tested along this work.

\subsection{Best bandwidth metric (bbandwidth)}

Since the end-to-end bandwidth of a path is a concave metric, it is equivalent to the bandwidth of the weakest of its links. In other words, the bandwidth of a path is given by the link with the narrowest bandwidth, in a similar way of a caudal traversing several funnels. Then, relying on this metric, a path selection algorithm should select the paths having the best bandwidth.

That is, given a path between two different nodes, $p\left(n_{o}, n_{d}\right)$, its cost is costminbw $(p)=\max _{l \in p}(\lfloor 1 /$ bandwidth $(l)\rfloor)$. Note that, the lower the path cost, the higher the possibility for the path to be selected.

For origin and destination nodes, $n_{o}$ and $n_{d}\left(n_{o} \neq n_{d}\right)$, let $P\left(n_{o}, n_{d}\right)$ be the set containing all possible paths starting in $n_{o}$ and arriving to $n_{d}$. With this metric, a path $p\left(n_{o}, n_{d}\right)$ (obviously $p \in P\left(n_{o}, n_{d}\right)$ ) would be chosen if costminbw $(p)=$ $\min _{k \in P}(\operatorname{costminbw}(k))$.

The interest of this metric is that bandwidth is the real bottleneck of community networks, especially when considering that the traffic of client nodes is concentrated in backbone links. In a typical scenario, a multi-radio node can have three antennas with a beam-width of $120^{\circ}$ for 87 client nodes, and two backbone point-to-point links. So, these two links have to manage the traffic for all client nodes plus additional traffic from other nodes if it belongs to the preferred 
path. Even worse, the traffic of these client nodes is typically concentrated in one of the two links because traffic is usually addressed to the same destinations, usually those providing public Internet access.

This metric has two main drawbacks. The first one is how to obtain a real time measure of the bandwidth of a link. To have an idea of the bandwidth of a link we have tried probing techniques which seems to provide good enough figures with a reduced overhead. Note that since we are talking about a infrastructure mode network with static nodes in house roofs, overhead can be tuned with the time between bandwidth samples. Changes are not so dynamic as in the case of mobile ad hoc networks.

The other drawback is that it does not consider the length or the latency of the path. In the experiments we observed a great number of equal cost paths as it is discussed in Section 6 . The number of hops from a node to another is the average of the hops of all found best-cost paths connecting them. The consequence is that the average number of hops is increased considerably.

\subsection{Number of hops (nhops)}

Hop count is the most widely used routing metric nowadays. OSPF automatically assigns a cost to each network interface based upon the bandwidth achievable by the underlying technology. If we call tbandwidth to this maximum theoretical bandwidth, the formula for the cost for a network interface, and consequently for a link, is $\left\lfloor 10^{8} /\right.$ tbandwidth $\rfloor$ if tbandwidth $\leq 10^{8}$ and 1 otherwise. The value $10^{8} \mathrm{bps}$ was a reference bandwidth in times of Fast Ethernet technology. Nowadays, this value can be updated, but for our study case it is not required since wireless links will typically fail to achieve this upper bandwidth bound.

OSPF implementations commonly assign the same cost to all wireless network interfaces and, consequently, this metric is reduced to the number of hops along the path. In fact, the cost of a path is obtained by adding the cost of each of its links, and so, shorter paths are the preferred ones. It could be seen as if latency were the most important aspect in this case.

To express this metric, we use a function, length $(p)$, returning the number of links (or hops) in a path $p$. Therefore, given two different nodes $n_{o}$ and $n_{d}$, let $P\left(n_{o}, n_{d}\right)$ be the set containing all possible paths starting in $n_{o}$ and arriving to $n_{d}$. A path selection algorithm using this metric, will give preference to a path $p\left(n_{o}, n_{d}\right) \in P\left(n_{o}, n_{d}\right)$ whenever length $(p)=\min _{k \in P}($ length $(k))$.

Notice that, although current OSPF implementation's metric is acceptable for wired networks where network devices usually have a stable bandwidth with very low error rates, it has bad consequences in the case of wireless networks due to the real-time variability of the links' quality.

\subsection{Real cost addition metric (addRC)}

In this case we suppose that links' bandwidth are measured in the same way as for the bbandwidth metric, being these estimated bandwidths the ones used instead of the adminis- trative values used in OSPF implementations.

For a directional link $l\left(n_{o}, n_{d}\right)$, let us suppose that the function bandwidth $(l)$ returns the measured bandwidth from $n_{o}$ to $n_{d}$ if a direct link between these two nodes exists, or 0 otherwise. In this metric, the cost of this link is $\left\lfloor 10^{8} /\right.$ bandwidth $\left.(l)\right\rfloor$. Finally, the cost of a path $p\left(n_{0}, n_{d}\right)$ is the addition of the cost of each of the links composing the path; that is, $\operatorname{costaddbw}(p)=$ $\sum_{l \in p}\left\lfloor 10^{8} /\right.$ bandwidth $\left.(l)\right\rfloor$. Note that the cost concept in each of the routing path metric is different, whereas in all, the lower the cost, the better is the link or the path.

For two nodes $n_{o}$ and $n_{d}$, a path selection algorithm using this metric would prefer those paths connecting from $n_{o}$ to $n_{d}$ whose cost is the minimal. That is, let be $P\left(n_{o}, n_{d}\right)$ the set of all possible paths between these (different) nodes. A path $p \in P$ is better for this metric if $\operatorname{costaddbw}(p)=$ $\min _{k \in P}(\operatorname{costaddbw}(k))$.

\subsection{Shortest widest path metric (shortbbandw)}

This path metric extends the bbandwidth one presented in Subsection 4.2 by selecting those paths with a lower hop count among the paths with the highest end-to-end bandwidth [1].

With this metric, the cost of a path $p$, which we call costshortbb( $p)$ is an array with two elements, the bandwidth of the path as stated in the bbandwidth metric (i.e. costmbw $(p)$ ), and the number of hops, length $(p)$ as in nhops metric. When comparing two paths, first it is compared their bandwidth and if they are equal, then is compared the number of hops. That is, for origin and destination nodes, $n_{o}$ and $n_{d}\left(n_{o} \neq n_{d}\right)$, let be $p\left(n_{o}, n_{d}\right)$ and $k\left(n_{o}, n_{d}\right)$ two different paths connecting them. With the shortbbandw metric, path $p$ is better than $k$ if, and only if, $(\operatorname{costmbw}(p)<\operatorname{costmbw}(k)) \vee$ $((\operatorname{costmbw}(p)=$ costmbw $(k)) \wedge$ length $(p)<$ length $(k)))$.

The definition of this path metric can be misleading because it is a two-dimensional metric. Multidimensional metrics are rarely used in practice due to their high computation cost. However in [1] it is shown that the algorithm used in OSPF can be adapted to apply this metric with similar computation effort.

The idea behind this metric is to use only the shortest paths with the best bandwidth in order to improve latency and jitter in transport or application layers. In preliminary simulations we observed that, if coarse bandwidth measurements were used (for instance, assigning bandwidth as the 802.11 modulation negotiation does), there would be a lot of routes with optimal bandwidth, but few of them would be disjoint. This metric would prevent such problem.

\section{PERFORMANCE METRICS CRITERIA}

In this section a description of the variables used for performance evaluation is provided, given that the result of the path metrics can be analysed from different points of view. The performance variables will be used to compare the different path selection algorithms in Section 6. The chosen metrics are the following: 
- Bandwidth. We take bandwidth of a path as the minimum bandwidth of its links. This bandwidth is supposed to be the capacity of the path without traffic in the network and considering that all resources are available just for this path. This values indicates the maximum bandwidth each route could reach. However, it does not consider other factors, such as the concentration of routes in few links, or the latency.

If there is more than one best-cost path connecting two nodes, the bandwidth is the aggregation; but if the paths are not disjoint, it is taken into account that the shared links can limit the aggregated bandwidth.

- Number of hops. It is the number of links in a given path. Since the number of hops is usually related to latency, having a low hop count is, in general, desirable. However, this variable does not directly refer to the bandwidth of the path nor to the concentration of routes.

If there are more than one best-cost path connecting two nodes, the number of hops is computed as the average number of hops of these paths regardless of whether they are disjoint or not.

- Bandwidth distribution function. This is the bandwidth distribution function for all paths. This function is interesting mainly to show the differences between the nhops metric, and the other path metrics, especially when dealing with low bandwidth routes. This can result in unconnected parts of a network due to routing decisions. It must be noted that nowadays, bandwidths lower than $1 \mathrm{Mbps}$ are only useful for what is known as ping networks.

\section{RESULTS}

Three guifi.net zones have been simulated in order to check whether the results are dependent on each particular topology. For each zone, the routes of each node to nodes offering public Internet access (sink nodes) are calculated using the four different proposed metrics. The topologies can be seen in Figures 2, 4, and 6. In these figures, green spots are the sink nodes and the thickness of links is proportional to its bandwidth. Each zone is described in terms of parameters such as the extension, number of nodes and links, or the amount of sink nodes.

The average routes' bandwidth and number of hops for the three zones can be seen respectively in Figures 8 and 9. Also, for each zone it is shown the routes' bandwidth distribution function obtained for each particular metric; Figure 10 shows the distribution for the three zones. As can be seen, nhops metric provides the worst bandwidth since it includes also the cases where bad performing links are involved. The metric bbandwidth gets the best bandwidth at the cost of higher number of hops, while shortbbandw improves bandwidth with a number of hops not much larger than with nhops.

\subsection{La Plana region in Castellón}

This area comprises the following guifi.net zones: Castellón, Almazora, Burriana, Vila-real, Benicàssim and Betxí. This topology represents an area of about $640 \mathrm{~km}^{2}(20 \mathrm{~km} \times 32$

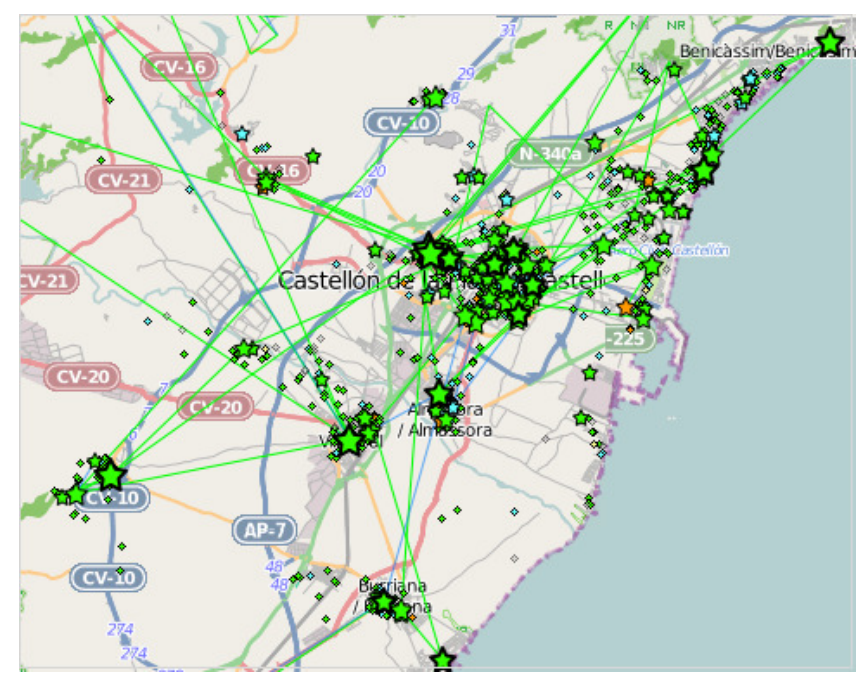

Figure 1: The guifi.net map of La Plana de Castellón region.

$\mathrm{km})$. The parameters simulated for this zone are the following:

- Destination nodes: 4 .

- Number of routing nodes: 58. Total number of nodes: 1750.

- Number of backbone bidirectional links: 86 .

- Best, worst, average and standard deviation of links' bandwidth: 76, 0.08, 50.9, and 19 Mbps respectively.

A capture of guifi.net map of this zone can be seen in Figure 1 .

Figure 3 shows the routes' bandwidth distribution function for this zone. For each metric, it shows how many routes have a bandwidth inferior or equal to a given value. In the figure it can be seen that the best distribution corresponds to the bbandwidth metric. It can be seen also that all metrics based on real bandwidth values largely outperforms the nhops metric for routes below $20 \mathrm{Mbps}$. For community networks this ensures that weak links are only used if there is no other choice or as backup links.

\subsection{Osona region in Barcelone}

This region comprises the following towns near Barcelona in Spain: Gurb, Vic, Sant Bartomeu, Manlleu, Roda de Ter and Voltreganés. This topology represents a total area of about $196 \mathrm{~km}^{2}(14 \mathrm{~km} \times 14 \mathrm{~km})$. The parameters simulated for this zone are the following:

- Destination nodes: 5 .

- Number of multirradio nodes: 74. Total number of nodes: 3680.

- Number of backbone bidirectional links: 176. 


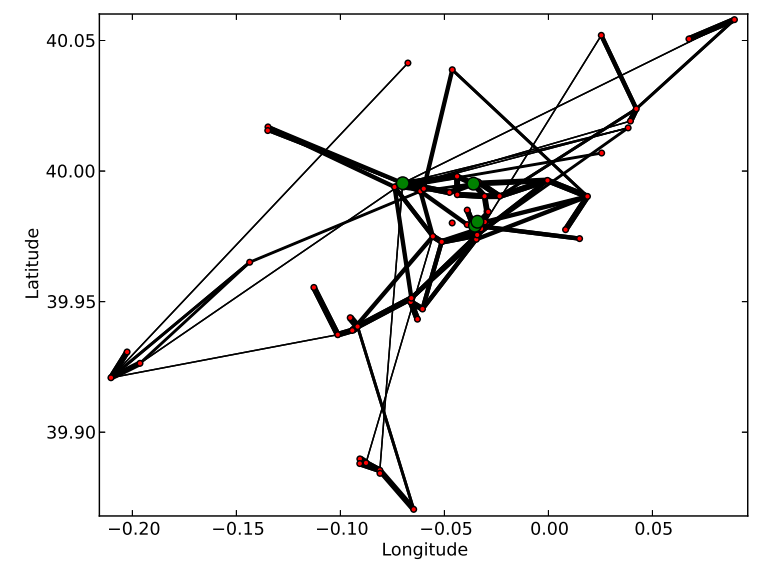

Figure 2: Topology extracted from guifi.net for the La Plana de Castellón region. Area of $20 \times 32 \mathrm{~km}^{2}$.

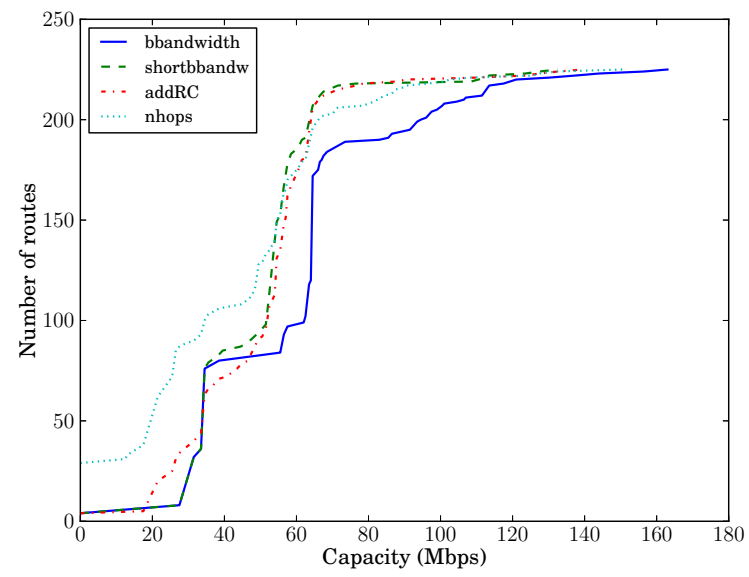

Figure 3: Distribution function of the bandwidths. La Plana de Castellón area.

- Best, worst, average and standard deviation of links' bandwidth: 77, 0.08, 43.3, $22.8 \mathrm{Mbps}$.

The topology of this area can be seen in Figure 4. The bandwidth distribution function is shown in Figure 5 . In this case the metrics based in real bandwidth values shows and important benefit when compared to the nhops metric, especially for routes below 20 Mbps.

\subsection{Barcelona city and Baix Llobregat region}

This region comprises cities of Barcelonés and Baix Llobregat. Overall it covers an area of about $493 \mathrm{~km}^{2}(17 \mathrm{~km} \times 29$ $\mathrm{km})$. The parameters simulated for this zone:

- Destination nodes: 5 .

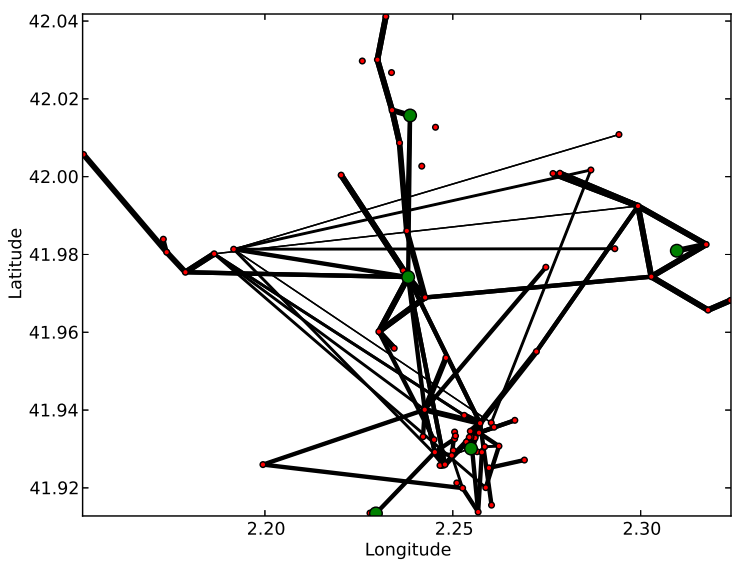

Figure 4: Topology extracted from guifi.net for the Gurb region. Area of $14 \times 14 \mathrm{~km}^{2}$.

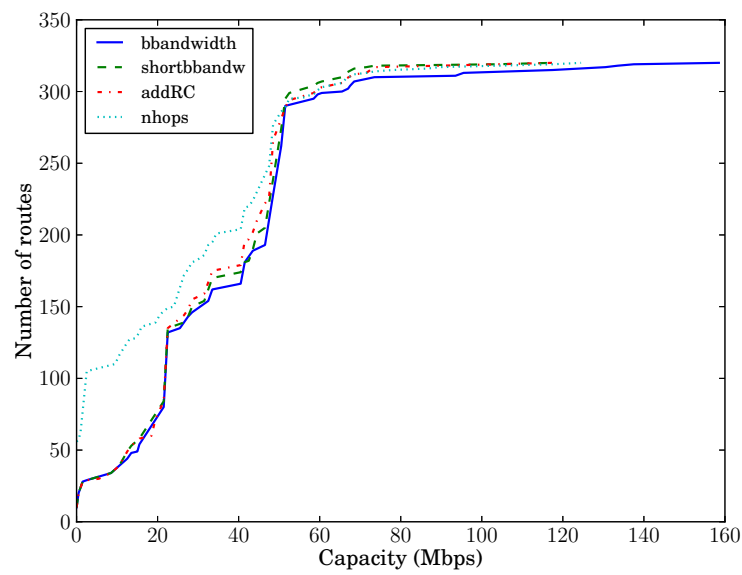

Figure 5: Distribution function of bandwidths. Osona region.

- Number of multirradio nodes: 162. Total number of nodes: 1718.

- Number of backbone bidirectional links: 298.

- Best, worst, average and standard deviation of links' bandwidth: 77, 0.08, 62.2, 14 Mbps.

Figure 7 shows the routes' bandwidth distribution function for the metropolitan Barcelona area. This figure has different profile to those of the other two areas. It is explained by the particularity that can be seen in its topology (Figure 6). On the left part of this figure there is a little cloud of nodes connected by two weak links to the main cloud and all sink nodes are in the main cloud. Consequently there is a reduced bandwidth for all routes between these two groups. 


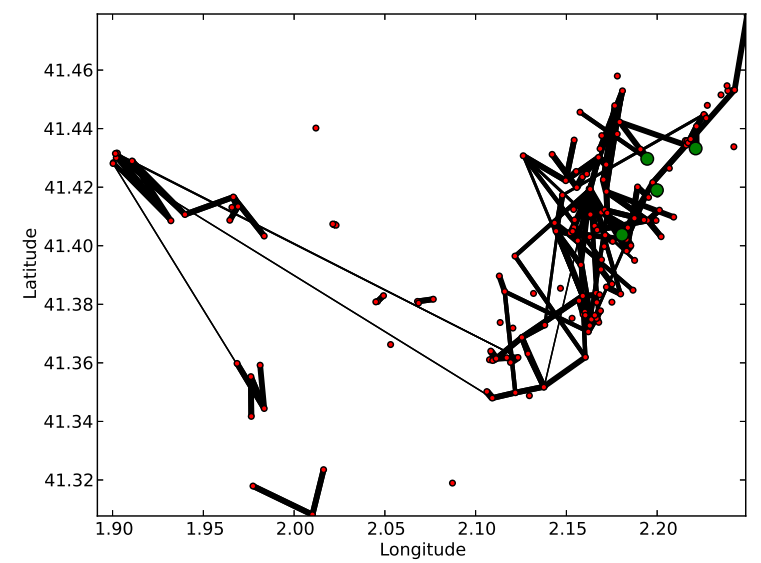

Figure 6: Topology extracted from guifi.net for the Barcelona region. Area of $17 \times 29 \mathrm{~km}^{2}$.

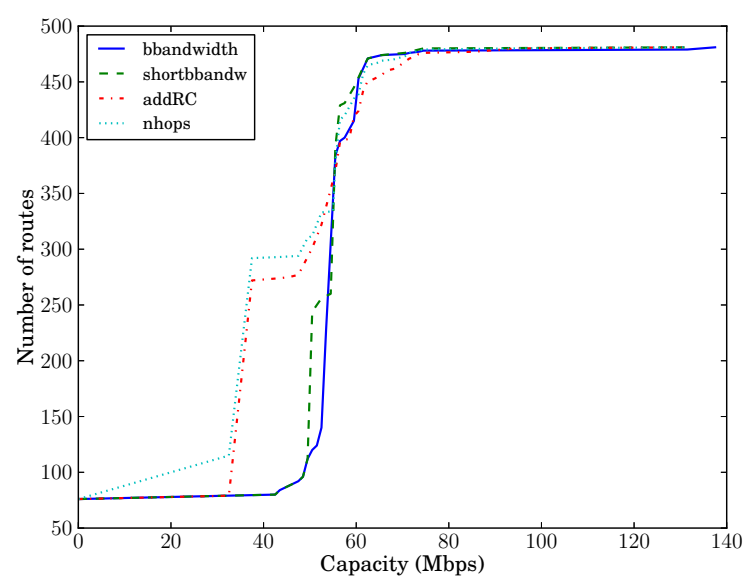

Figure 7: Distribution function of bandwidths. Barcelone and Baix Llobregat region.

\subsection{Analysis of the results}

Figure 10 shows that the nhops metric doubles the amount of routes having less bandwidth than $20 \mathrm{Mbps}$. It is even worse in the case of La Plana de Castellón (Figure 3).

We computed the average number of disjoint best-cost multipath routes obtained with the different metrics and the results were quite similar i.e., hardly more than one disjoint paths per route. It seems that this factor is not so important in real topologies as we thought, given that the average number of backbone links per router is of about 3.5. This is the limit for alternative disjoint routes. Probably the only way to improve this factor is applying suboptimal multipath routing.

As expected, the bbandwidth metric improves the network capacity. However, we observed that this metric also pro-

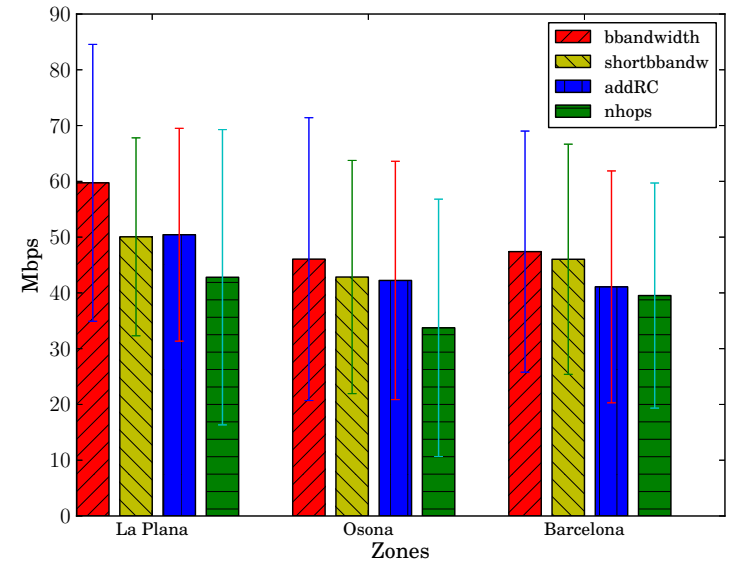

Figure 8: Average bandwidths provided with the different metrics in the three evaluated zones.

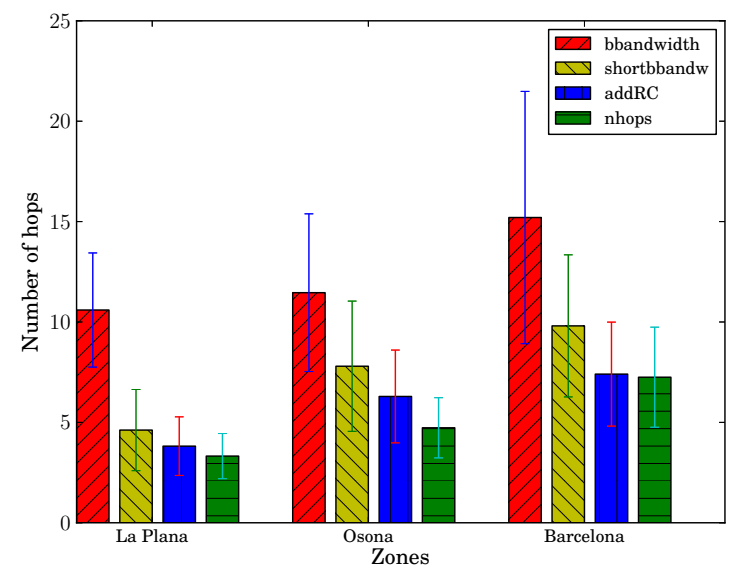

Figure 9: Average number of hops obtained with different metrics in the three evaluated zones.

vides an important number of overlapping best-cost routes. The average number of equivalent routes with bbandwidth metric is around 28 and when selecting the nhops metric it is of 1.5. If traffic balance is based on TCP connexions, the network load could be distributed in a better way, and in the worst case, the performance would be close to that of using only disjoint routes due to TCP congestion windows. To the best of our knowledge there are no real implementations of multipath routing applying per packet traffic balance.

The shortbbandw metric makes a better selection of equivalent paths but then, a reduced bandwidth is obtained. We proposed this metric in order to reduce the number of bestcost overlapping paths. Figure 10 shows a bandwidth distribution close to that of the bbandwidth metric. In Figure 3 it can be seen that, in one of the regions (la Plana de Castellón) there is a significant bandwidth difference between bbandwidth and shortbbandw metrics, but in the two 


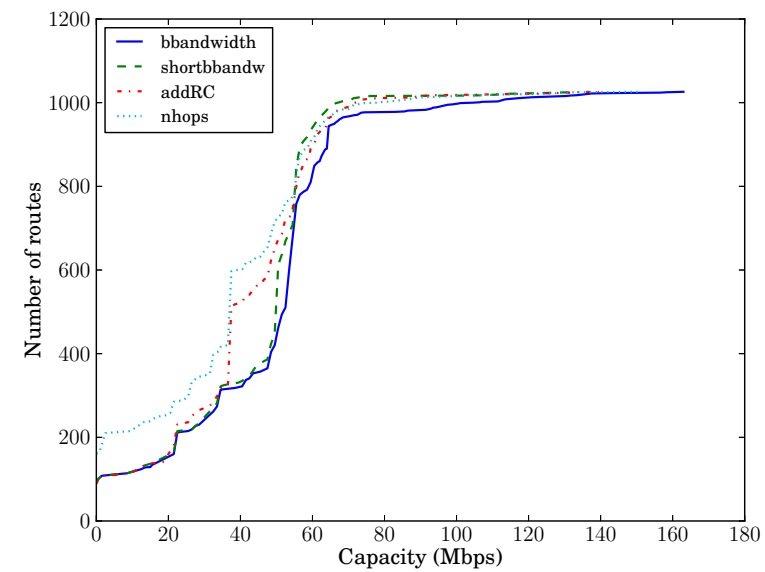

Figure 10: Bandwidth distribution function accumulating the routes of the three regions.

other zones the average bandwidth obtained with the two metrics is quite similar.

Regarding the average number of hops, the nhops metric is obviously the best one. Nevertheless, what can be surprising is that the bbandwidth metric almost doubles the number of hops. The reason is that, in this case, there are a lot of equivalent routes with different lengths.

A solution to reduce the number of routes would be to compute the cost more accurately than OSPF does, so the number of links with the same cost would be reduced and, consequently, the number of non-disjoint alternative paths. Another simple solution may consists in incrementing the granularity in the cost formula (for instance taking into account some decimals). If more accurate cost values are used, fewer equivalent routes would be found, and consequently the average number of hops per route would be reduced.

\section{CONCLUSIONS AND FUTURE WORK}

In this work we showed that current implementations of routing protocols such as OSPF have performance problems in real wireless network environments. On the one hand, these protocols make wrong decisions which cause some parts of the network to be under-utilized. On the other hand, better bandwidth estimations could be used to improve routing decisions. The results of the three tested metrics using real bandwidth values (bbandwidth, shortbbanw and $a d d R C$ ) showed an important improvement with respect to the metric representing just the number of hops; especially for the worst connected parts of the networks.

We also shown that the metrics we have compared have similar results with regard to alternative disjoint best-cost routes. Experimental results demonstrate that path selection based on bandwidth maximization provides the best capacity but also generates a great number of overlapping best-cost routes.

As a consequence of these results we are working in the con- text of large WCN to verify the impact of using overlapping multi-path routes, reviewing probing methods to measure the links' bandwidth with limited intrusion, and finally designing an implementation of all these mechanisms on real devices.

\section{ACKNOWLEDGMENTS}

This work has been supported by project P1·1A2010-13 from the Universitat Jaume I, the project TIN2011-27543-C03-01 from "Ministerio de Ciencia e Innovación de España" and also by the ERDF of the European Union.

\section{REFERENCES}

[1] G. Apostolopoulos, D. Williams, S. Kamat, R. Guerin, A. Orda, T. Przygienda, et al. Qos routing mechanisms and ospf extensions. Technical report, RFC 2676, August, 1999.

[2] R. Baumann, S. Heimlicher, M. Strasser, and A. Weibel. A survey on routing metrics. TIK Report, 262, 2007.

[3] L. Cerda-Alabern. On the topology characterization of guifi.net. 2013 IEEE 9th International Conference on Wireless and Mobile Computing, Networking and Communications (WiMob), 0:389-396, 2012.

[4] R. Draves, J. Padhye, and B. Zill. Comparison of routing metrics for static multi-hop wireless networks. In In ACM SIGCOMM, pages 133-144, 2004.

[5] G. Parissidis, M. Karaliopoulos, R. Baumann, T. Spyropoulos, and B. Plattner. Routing metrics for wireless mesh networks. In S. Misra, S. Misra, and I. Woungang, editors, Guide to Wireless Mesh Networks, Computer Communications and Networks, pages 199-230. Springer London, 2009.

[6] R. Prasad, C. Dovrolis, M. Murray, and K. Claffy. Bandwidth estimation: metrics, measurement techniques, and tools. Network, IEEE, 17(6):27-35, 2003.

[7] E. M. Royer and C.-K. Toh. A review of current routing protocols for ad hoc mobile wireless networks. Personal Communications, IEEE, 6(2):46-55, 1999.

[8] D. Seither, A. Konig, and M. Hollick. Routing performance of wireless mesh networks: A practical evaluation of batman advanced. In Local Computer Networks (LCN), 2011 IEEE 36th Conference on, pages 897-904. IEEE, 2011.

[9] B. Sen, L. Libman, X. Zhao, and S. Jha. An end-to-end delay metric for multi-rate wireless mesh networks with cooperative retransmission. In Local Computer Networks (LCN), 2013 IEEE 38th Conference on. IEEE, 2013.

[10] R. K. Sheshadri and D. Koutsonikolas. An experimental study of routing metrics in $802.11 \mathrm{n}$ wireless mesh networks. IEEE Transactions on Mobile Computing, 99(PrePrints):1, 2014.

[11] Z. Wang and J. Crowcroft. Quality-of-service routing for supporting multimedia applications. Selected Areas in Communications, IEEE Journal on, 14(7):1228-1234, 1996. 